\title{
Very massive stars: a metallicity-dependent upper-mass limit, slow winds, and the self-enrichment of globular clusters
}

\author{
Jorick S. Vink
}

\begin{abstract}
Armagh Observatory and Planetarium, College Hill, Armagh BT61 9DG, Ireland
e-mail: jsv@arm.ac.uk
\end{abstract}

Received 5 February 2018 / Accepted 21 March 2018

\begin{abstract}
One of the key questions in Astrophysics concerns the issue of whether there exists an upper-mass limit to stars, and if so, what physical mechanism sets this limit? The answer to this question might also determine if the upper-mass limit is metallicity $(Z)$ dependent. We argue that mass loss by radiation-driven winds mediated by line opacity is one of the prime candidates setting the upper-mass limit. We present mass-loss predictions $\left(\dot{M}_{\text {wind }}\right)$ from Monte Carlo radiative transfer models for relatively cool $\left(T_{\text {eff }}=15 \mathrm{kK}\right)$ very inflated massive stars (VMS) with large Eddington $\Gamma$ factors in the mass range $10^{2}-10^{3} M_{\odot}$ as a function of metallicity down to $1 / 100 Z / Z_{\odot}$. We employed a hydrodynamic version of our Monte Carlo method, allowing us to predict the rate of mass loss $\left(\dot{M}_{\text {wind }}\right)$ and the terminal wind velocity $\left(v_{\infty}\right)$ simultaneously. Interestingly, we find wind terminal velocities $\left(v_{\infty}\right)$ that are low $\left(100-500 \mathrm{~km} \mathrm{~s}^{-1}\right.$ ) over a wide Z-range, and we propose that the slow winds from VMS are an important source of self-enrichment in globular clusters. We also find mass-loss rates $\left(\dot{M}_{\text {wind }}\right)$, exceeding the typical mass-accretion rate $\left(\dot{M}_{\text {accr }}\right)$ of $10^{-3} M_{\odot} \mathrm{yr}^{-1}$ during massive-star formation. We have expressed our mass-loss predictions as a function of mass and $Z$, finding $\log \dot{M}=-9.13+2.1 \log \left(M / M_{\odot}\right)+0.74 \log \left(Z / Z_{\odot}\right)\left(M_{\odot} / \mathrm{yr}\right)$. Even if stellar winds do not directly halt \& reverse mass accretion during star formation, if the most massive stars form by stellar mergers, stellar wind mass loss may dominate over the rate at which stellar growth takes place. We therefore argue that the upper-mass limit is effectively $Z$-dependent due to the nature of radiation-driven winds. This has dramatic consequences for the most luminous supernovae, gamma-ray bursts, and other black hole formation scenarios at different Cosmic epochs.
\end{abstract}

Key words. stars: early-type - stars: mass-loss - globular clusters: general - stars: formation - stars: winds, outflows

\section{Introduction}

We present mass loss-predictions for very massive stars (VMS) in the $10^{2}-10^{3} M_{\odot}$ range, which may also provide useful insights for winds from supermassive stars (SMS) above $10^{3} M_{\odot}$ range that may exist in the dense centres of globular clusters (e.g. Portegies Zwart et al. 2004). SMS have been suggested to be responsible for the observed anti-correlations in stellar abundances (between $\mathrm{Na}$ and $\mathrm{O} ; \mathrm{Mg}$ and $\mathrm{Al}$ ) of low-mass stars in globular clusters (Denissenkov \& Hartwick 2014; Gieles et al. 2018). Here we propose our predicted slow winds from VMS as a source of internal pollution of Globular Clusters as an alternative.

Until 2010, most astronomers thought the stellar initial mass function (IMF) had an upper limit in the range 100-150 $M_{\odot}$ (Weidner \& Kroupa 2004; Figer 2005) and for this reason early investigations of potential polluters of Globular clusters by Prantzos \& Charbonnel (2006) only included stars up to $100 M_{\odot}$ in their analysis of possible pollution by the winds from massive stars as an alternative to massive asymptotic giant branch (AGB) stars. We have recently discovered in the context of the VLT-Flames Tarantula Survey (VFTS; Evans et al. 2011; Vink et al. 2017) that the number of massive stars above $30 M_{\odot}$ is significantly larger than expected from a Salpeter IMF (Schneider et al. 2018). But there is more: within the same VFTS survey we found evidence for an upturn in the mass-loss rates of VMS above $\sim 100 M_{\odot}$ (Bestenlehner et al. 2014), as predicted (Vink et al. 2011). These stars are identified as WNh stars (Crowther et al. 2010; Gräfener et al. 2011; Martins 2015) with a nitrogen-enhanced Wolf-Rayet appearance, but still with hydrogen $(\mathrm{H})$ present, as expected for H-burning main sequence stars. Thus, in contrast to the hypothetical SMS, we can be sure that VMS exist in nature.

Regarding globular clusters, one of the main problems with most of the proposed (AGB or massive star) self-enrichment sources from nucleo-synthesis is the so-called mass budget problem (Bastian \& Lardo 2018). However, given the discovered excess of VMS, enhancing the kinetic wind energy and momentum by at least a factor of five (Schneider et al. 2018), as well as the enhanced stellar wind strength from these very massive stars, VMS may be both plentiful and individually powerful enough to provide the required amount of mass loss to overcome the mass-budget problem, naturally becoming the main contender for being the source of the chemical pollution of globular clusters. So far however, the most fundamental opposition to the winds of (very) massive stars has been the property of their fast (2000-3000 $\mathrm{km} \mathrm{s}^{-1}$ ) outflow speeds, significantly larger than the potential wells of globular clusters would allow (e.g. Decressin et al. 2007; de Mink et al. 2009; Gieles et al. 2018). However, here we predict slow winds from VMS for cool $(\simeq 15 \mathrm{kK})$ and inflated VMS, allowing the winds from VMS to be the most natural source for the self-enrichment of globular clusters.

One of the key questions in astrophysics concerns the question of whether there exists an upper mass limit to stars, and what physical mechanism may set such an upper limit. Prior to the inclusion of the OPAL opacities in stellar structure models, even at solar $Z$ it was possible to construct stellar models for stars with $10^{6} M_{\odot}$ or more (e.g. Kato 1986), but this limit has dropped 
to $10^{3} M_{\odot}$ over the last decade (Ishii et al. 1999; Yungelson et al. 2008). This still does not mean that stars of $10^{3} M_{\odot}$ will actually form in nature at solar $Z$, as the actual upper limit may be dependent on various feedback effects (Krumholz 2015; Tanaka et al. 2017) of which stellar winds is one of them.

For many decades it appeared almost impossible to form massive stars without the need to resort to stellar mergers, as radiation pressure on dust grains could reverse the infalling material - limiting the stellar mass to a maximum value as low as $\sim 10 M_{\odot}$ (e.g. Larson \& Starrfield 1971; Wolfire \& Cassinelli 1987). However, in these early models the accretion was assumed to be spherically symmetric - an unlikely scenario in nature. Indeed, more recent multi-D simulations (Yorke \& Sonnhalter 2002; Krumholz et al. 2009; Kuiper et al. 2010; Rosen et al. 2016) indicate there is no fundamental problem in growing (very) massive stars via equatorial accretion disks. The only limiting factor seems to be the amount of material initially available for the hydrodynamical simulations (Krumholz 2015).

We note that the star-formation simulations do not actually resolve the innermost grid-point, and it is stellar physics that ultimately determines the fate of the object. For example, the above-mentioned numerical simulations only include dust opacity, but do not include the line opacity of atomic gas - known to be the dominant opacity source in mass-loss computations for massive stars, since the early 1970s (Lucy \& Solomon 1970; Castor et al. 1975 (CAK); Pauldrach et al. 1986; Vink et al. 2000; Müller \& Vink 2008; Krtička et al. 2016).

Tanaka et al. (2017) recently showed that given typical massaccretion rates of order $10^{-3} M_{\odot} \mathrm{yr}^{-1}$, feedback mechanisms need to be of the same order of magnitude as this high massaccretion rate to significantly affect the maximum stellar mass. They considered mass-loss rates from stellar winds for hot stars $(\simeq 50 \mathrm{kK})$, and estimated these to be too low. However, VMS are expected to be "bloated" (Hosokawa \& Omukai 2009) before they reach the zero-age main sequence (ZAMS), for instance, due to envelope inflation due to the Fe opacity bump (Ishii et al. 1999; Gräfener et al. 2012). We should therefore analyse mass-loss rates at lower effective temperatures $\left(T_{\text {eff }}=15 \mathrm{kK}\right)$ below the so-called wind bi-stability jump (Pauldrach \& Puls 1990; Vink et al. 1999) where, as we show in this paper, the rates can reach the same order of magnitude as the typically assumed mass-accretion rate of $10^{-3} M_{\odot} \mathrm{yr}^{-1}$. This suggests that metallicity-dependent stellar winds ultimately cause the upper-mass limit to be $Z$ dependent.

Interestingly, the most massive stars may possibly form by stellar collisions between lower mass stars in dense clusters (e.g. Portegies Zwart et al. 2010; Banerjee et al. 2012). This scenario may possibly lead to the formation of $1000 M_{\odot}$ VMS if mass loss during stellar evolution were not important. However, Belkus et al. (2007); Yungelson et al. (2008); Pauldrach et al. (2012); Yusof et al. (2013) and Köhler et al. (2015) showed that the "effective" upper mass limit is expected to be far lower than $1000 M_{\odot}$ due to stellar wind mass loss at solar metallicity.

Here we investigate if the mass-loss rates of VMS in the $10^{2}-10^{3} M_{\odot}$ range are Z-dependent, arguing for a $Z$-dependent effective upper mass limit. VMS are observed to have masses up to 200-300 $M_{\odot}$, which appears to be the currently known empirical upper-mass limit (Crowther et al. 2010; Bestenlehner et al. 2014; Martins 2015). Such stars are no longer identified as O-type stars, but WNh stars (Wolf-Rayet stars with nitrogen and hydrogen), which simply form the extension of the main sequence to the highest known empirical stellar masses (Vink et al. 2015), forming a natural sequence of O stars, Of/WN transition stars, and the most massive WNh emission line stars due to higher \& higher mass loss. Although there is no need to invoke that these WNh stars are stellar mergers above the canonical Figer (2005) $150 M_{\odot}$ limit, it is of course well possible that mergers (either as dynamical collisions, or via binary evolution) could contribute to making the most massive stars.

VMS are key for correctly predicting the ionizing radiation of these hot stars, with major consequences for interpreting He II line emission at intermediate and high red-shifts as Population III stars (Cassata et al. 2013; Sobral et al. 2015), or VMS at low metallicity (Gräfener \& Vink 2015).

This paper is organized as follows. In Sect. 2, we briefly describe the Monte Carlo modelling, and the parameter space considered for this study (Sect. 3). The mass-loss predictions (Sect. 4) for cool VMS are followed by discussions on the relevance for massive star formation feedback and the uppermass limit (Sect. 5) and the self-enrichment of globular clusters (Sect. 6), before ending with a summary in Sect. 7 .

\section{Physical assumptions and Monte Carlo modelling}

In this paper we predict mass loss due to stationary stellar winds on the basis of the Castor et al. (1975) radiation-driven wind model, with modifications to allow for multiple-line scattering. The details of our approach - inspired by Abbott \& Lucy (1985) are given in Vink et al. (1999 and references therein). As in our previous computations of mass-loss rates for hotter VMS (Vink et al. 2011), we employ our dynamical approach (Müller \& Vink 2008, 2014; Muijres et al. 2012), in which we predict $\dot{M}, v_{\infty}$ and the wind structure parameter $\beta$ simultaneously.

The underlying model atmosphere code ISA-WIND (de Koter et al. 1993) computes the chemical elements H, He, C, N, O, $\mathrm{S}$, and $\mathrm{Si}$ explicitly in non-LTE. The iron-group elements are treated in the simplified approach of Schmutz (1991). Tests were performed in which we treated Fe explicitly in non-LTE, but as this showed only very small differences with respect to the simplified models, we kept treating Fe in the approximate way. The Kurucz \& Bell (1995) line list includes million of lines, of which we selected the strongest $10^{5}$ transitions of the first 30 elements in the periodic table.

We have assumed the winds to be spherically symmetric and homogeneous. For hot massive stars in the canonical mass range, wind clumping is known to have a profound effect (Hillier 1991), leading to a downwards adjustment of empirical mass-loss rates, by a factor of $\simeq 3$ (Moffat \& Robert 1994; Puls et al. 2008; Hamann et al. 2008; Ramírez-Agudelo et al. 2017).

Wind clumping may also have a theoretical effect on the radiative driving. Furthermore, given that VMS are relatively close to the Eddington limit there could be additional physics resulting in the development of porous structures (van Marle et al. 2008; Gräfener et al. 2012; Jiang et al. 2015). The issue of porosity on mass-loss rate predictions for O-type stars was studied by Muijres et al. (2011), where it was found that whilst the impact on $\dot{M}$ can be large for certain clumping prescriptions, the overall conclusion for moderate clumping and porosity is that it does not change the mass-loss predictions substantially. In the present set of computations we do therefore not account for wind clumping.

Proximity to the Eddington limit may also lead to eruptive mass loss in luminous blue variable (LBV) Eta Car type giant eruptions (e.g. Shaviv 2000; Smith \& Owocki 2006; 
Owocki 2015). This may lead to additional mass loss, which may be less dependent on metallicity (although due to the Fe opacity bump the metallicity may still play a role). We note however that the model-independent transition mass-loss rate of Vink \& Gräfener (2012), which allowed a calibration of stationary massloss rates, showed that giant eruptions are not required to offset the lower mass-loss rates from clumped stationary winds. However, if LBV-type giant eruptions do occur sufficiently frequently (and vigorously), they might provide an additional avenue for mass loss, on top of what is predicted in this paper.

\section{Parameter space}

We managed to converge models up to $900 M_{\odot}$ within the Müller \& Vink (2008) dynamical framework, to estimate the mass-loss rates and wind velocities of VMS. For a range of masses, stellar luminosities were derived from the mass-luminosity relation of Gräfener et al. 2011, forming the basis of our grid. We added a few additional models where the stellar mass was varied with respect to the standard luminosity value, thus changing the $M-L$ ratio, and thus the Eddington $\Gamma$ value (see the bottom of Table 1). We note that we refer to the Eddington factor for electron scattering only, but for a more extensive discussion on the total opacity see Vink et al. 2011.

The effective temperature sets the ionization stratification in the atmosphere and determines which lines are most active in driving the wind. As a result, $T_{\text {eff }}$ affects the predicted massloss rate. Here, we fix $T_{\text {eff }}$ to $15000 \mathrm{~K}$. The reason for these cool temperatures is envelope inflation, which is thought to occur both during pre-main sequence (PMS) and post-main sequence evolution (Tanaka et al. 2017; Gräfener et al. 2012). We do not express the mass-loss rates as a function of $T_{\text {eff }}$, as radii are highly uncertain due to the likely clumped nature of inflated stellar envelopes (Gräfener et al. 2012).

Our grid has been constructed to predict the mass-loss behaviour as a function of $M$ (or $\Gamma$ via the $M-L$ relation) and $Z$, which are scaled to the solar values (Anders \& Grevesse 1989), and investigated down to $1 / 100 Z / Z_{\odot}$, as relevant for VMS in the present-day universe, as well as for globular cluster metallicities (see Sect. 6).

\section{Results}

Table 1 lists our mass-loss predictions. The initial stellar parameters are given in Cols. (1)-(5). The predicted wind terminal velocities, mass-loss rates, and wind acceleration parameter $\beta$ are listed in Cols. (6), (7), and (8). The predicted mass-loss rates are shown in Fig. 1, and the resulting terminal wind velocities are shown in Fig. 2. Different symbols are used to identify the different $Z$ ranges. For clarity, not all $Z$ models feature here, but only alternating $Z$ values from Table 1 have been plotted.

Figure 1 shows that $\dot{M}$ increases with $\Gamma$ and $Z$, as expected (Abbott 1982; Vink et al. 2001; Kudritzki 2002). Generally, the predicted mass-loss rates are in good agreement with the nondynamically consistent metallicity-dependent mass-loss rates of Vink et al. (2000, 2001). We note however that the 2000/2001 recipe that was provided alongside the predicted rates in the form of an IDL routine bases its output on the location of the second bi-stability jump (Vink et al. 1999, Petrov et al. 2016). This means that where the 2000/2001 recipe indicates a location below the second bi-stability jump, it gives larger values (by a factor of a few) than predicted for a fixed value of $15000 \mathrm{~K}$. It is thus important to assess the temperature location of bi-stability

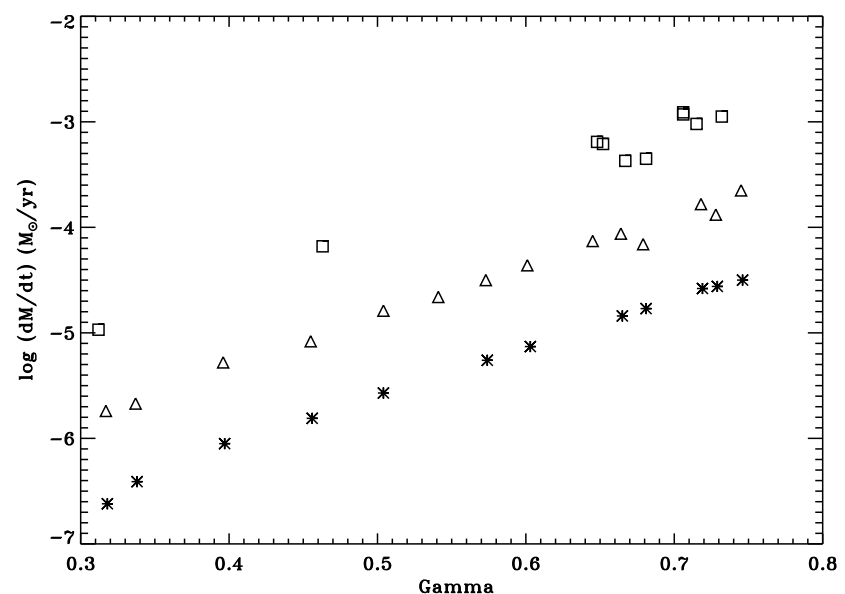

Fig. 1. Predicted mass-loss rates $\left(\dot{M}_{\text {wind }}\right)$ versus $\Gamma$ for solar metallicity models (open squares), models of $10 \% Z_{\odot}$ (open triangles) and $1 \%$ $Z_{\odot}$ (asterisks). The mass-loss rates show the expected linear drop with lower $Z$.

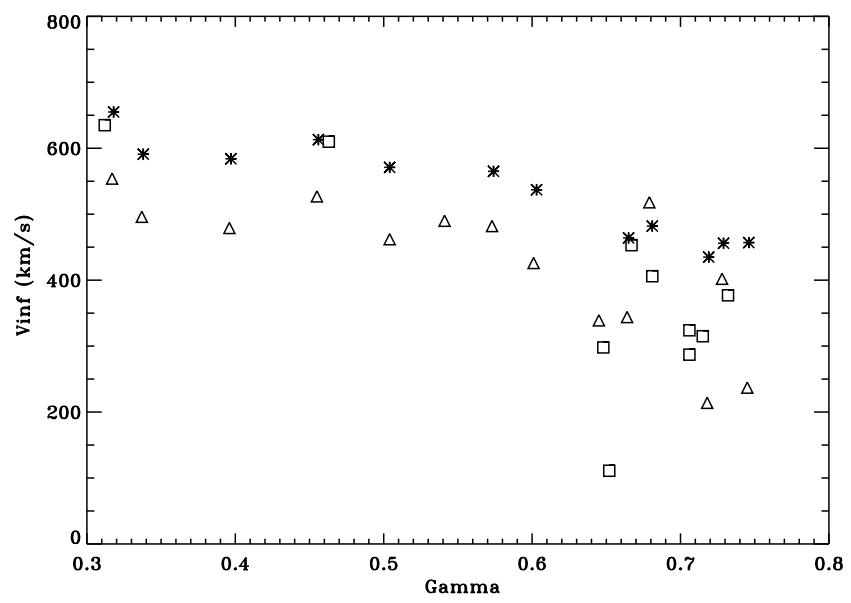

Fig. 2. Predicted wind velocity $\left(v_{\infty}\right)$ versus $\Gamma$ for solar metallicity models (open squares), models of $10 \% Z_{\odot}$ (open triangles), and $1 \% Z_{\odot}$ (asterisks). We note that the wind velocities do not show a linear drop with $Z$. The reason is explained in the main body text.

jumps before for example comparing them to observed values. This also shows that the true mass-loss rates of bloated VMS may be even higher than predicted in this paper (if the stars make it to cooler temperatures than considered here). Moreover, we note that at these relatively cool temperatures our models remain optically thin due to the large stellar radii, and kinks in the $\dot{M}$ versus $\Gamma$ relation which were present for hotter VMS models (Vink et al. 2011), are thus absent.

We now turn our attention to the wind velocity structure parameter, $\beta$, which describes how rapidly the wind accelerates. The predicted values of $\beta$ are given in column (8) of the table. $\beta$ values are mostly in the range $0.6-0.8$, in accordance with the models of Pauldrach et al. (1986), Müller \& Vink (2008), and Muijres et al. (2012).

Figure 2 shows relatively low terminal wind velocities in the range 400-600 $\mathrm{km} \mathrm{s}^{-1}$ for moderately high (0.3-0.6) Eddington models, going down to $100-200 \mathrm{~km} \mathrm{~s}^{-1}$ at even larger Eddington values. The main reason for these low values has little to do with metallicity (see below). The main reasons are (i) the low effective gravity \& escape velocity for these large stars, and the (ii) high mass-loss rates for these high $\Gamma$ objects instead. 
A\&A 615, A119 (2018)

Table 1. Mass-loss predictions for VMS with parameters from the Gräfener et al. (2011) $M-L$ relationship.

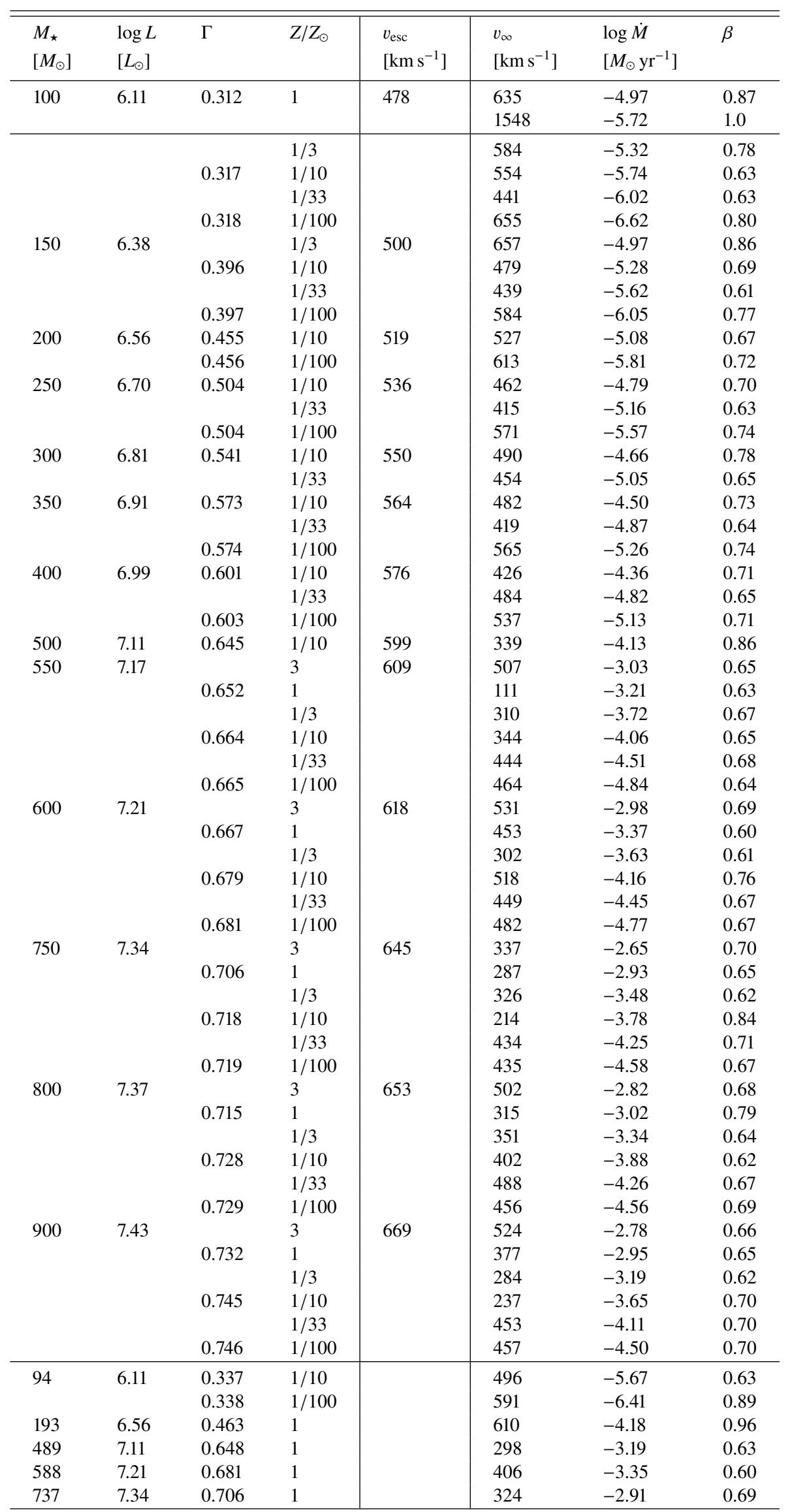

Notes. A few extra models are shown below the line at the bottom of the table. $T_{\text {eff }}$ is kept constant at $15000 \mathrm{~K}$. 
These slow winds are most relevant for a potential role of VMS for enriching Globular Clusters, as fast winds from normal O-stars might escape the potential well of the young Globular Cluster. These slow winds might also be relevant for the narrow He II line emission seen at intermediate (Cassata et al. 2013) and high (Sobral et al. 2015) redshift as discussed in Gräfener \& Vink (2015).

Interestingly, the wind terminal velocities do not display a linear drop with $Z$, as is the case for $\dot{M}$, shown in Fig. 1. Figure 2 shows that the triangles representing intermediate values of $10 \%$ $Z_{\odot}$ to be lower than both the high (solar) $Z$ squares, as well as the very low $1 \% Z_{\odot}$ asterisks. The reason for this non-linear behaviour is that there are two competing physical effects. The first one is the direct line driving effect: less efficient driving at low $Z$ leads to smaller terminal velocities. The second effect is that due to the lower $\dot{M}$ at lower $Z$, the lower density at the critical (sonic) point leads to a larger terminal wind velocity, as the driving of optically thick lines - relevant for the supersonic portion of the wind that determines the terminal wind velocity - is inversely proportional to the density. This means there must exist a minimum wind velocity for our objects. In our set of model calculations, the minimum wind velocities are reached at $Z / Z_{\odot}$ of $\simeq 1 / 33$, that is, at globular cluster metallicities of order $[\mathrm{Fe} / \mathrm{H}] \simeq-1.5$.

\subsection{Mass loss recipe for VMS as a function of metallicity}

In order to determine the dependence of the mass-loss rate on $M$ and $Z$ simultaneously, we perform multiple linear regression, finding

$\log \dot{M}=-9.13+2.1 \log \left(M / M_{\odot}\right)+0.74 \log \left(Z / Z_{\odot}\right)\left[M_{\odot} \mathrm{yr}^{-1}\right]$,

with a fitting error of $\sigma=0.09$. The formula was derived for the $Z$ range $\left(Z / Z_{\odot}\right)=1-10^{-2}$ and the mass range $100-900 M_{\odot}$. Extrapolation into the regime of SMS above $10^{3} M_{\odot}$ range is at potential users' own risk.

The mass-loss versus luminosity or Eddington $\Gamma$ relationship can be transformed using relevant mass-luminosity relationships (Gräfener et al. 2011).

\subsection{Comparison to observations and previous models}

It is not possible to directly compare our new predictions against observations or previous model predictions as models in this parameter range have not been calculated before. The mass-loss rates predicted here for $15 \mathrm{kK}$ are larger than those computed by Vink et al. (2011), with the main reason being the bi-stability jump (Vink et al. 1999). Terminal wind velocities in LBVs undergoing $S$ Doradus type variations are generally of the order of 100-500 $\mathrm{km} \mathrm{s}^{-1}$ (see Vink \& Gräfener 2012), lending empirical support for our wind predictions.

Gräfener \& Hamann (2008) computed optically thick wind models for hotter WNh stars as a function of $Z$, finding them to be lower than those of Vink et al. (2000, 2001). Also, the optically thin wind models by Pauldrach et al. (2012) gave lower mass-loss rates than Vink et al. $(2000,2001)$ for hot VMS in the hotter $(40-50 \mathrm{kK})$ range.

\section{The "effective" upper mass limit of stars}

For nucleo-synthesis of VMS (Woosley \& Heger 2015) and the associated (maximum) metallicity-dependent mass limits

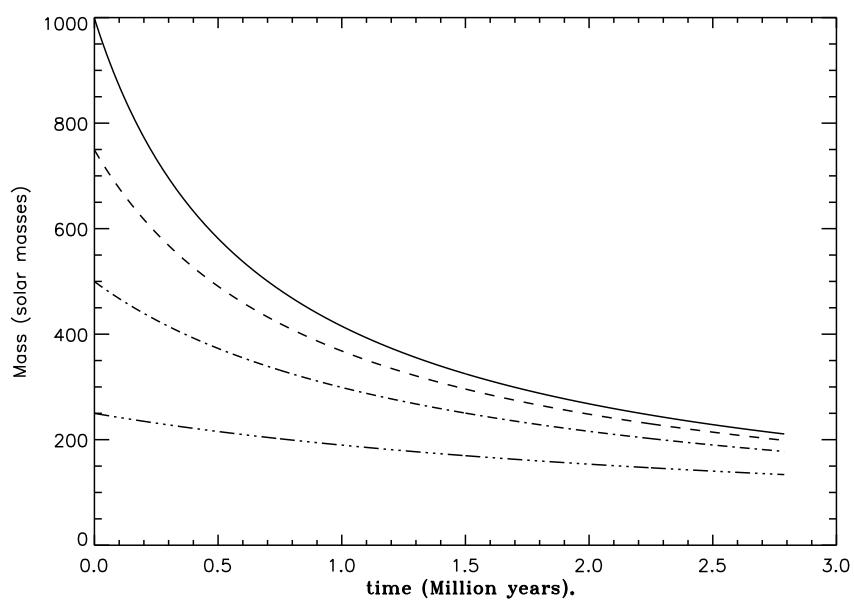

Fig. 3. "Effective" upper-mass limit at solar metallicity. The effective limit drops with time, as four different initial masses $(250,500,750$, $1000 M_{\odot}$ ) - represented by the four different lines - "converge" to very similar values. The figure also highlights that it is not possible to infer the value of the initial stellar upper-mass limit from present-day observations (unless one has the exact theoretical knowledge of the mass-loss history available).

of black holes (Eldridge \& Vink 2006; Belczynski et al. 2010), the important parameters are the maximum mass that star formation allows for as well as the subsequent mass-loss history. Whether the most massive stars form by core accretion, mergers, or coalescing stars in dense clusters, we argue that the most meaningful parameter is the effective uppermass limit for stars with different $Z$, that incorporates both star formation and early stellar evolution including stellar wind effects.

This effective upper-mass limit is set not only by $Z$, but also by the absolute value of the mass-dependent mass-loss rate, derived above. This radiation-driven mass-loss rate may already be relevant during the PMS evolution during massive star formation, or otherwise during the core-hydrogen main sequence evolution of merger products. The final result will depend on the exact physical mechanism that sets the mass-accretion rate during star formation, whether or not it is $Z$ dependent (de Marchi et al. 2011, but see Kalari \& Vink 2015).

However, whilst it not yet established if the mass-accretion rate is $Z$ dependent, we may at least conclude that the effective upper-mass limit is $Z$ dependent, with a higher effective uppermass limit for lower $Z$ galaxies. The quantitative value of the upper-mass limit - for each $Z$ - will however depend on the mass-dependent mass-loss rate.

During stellar evolution (and/or formation) the stellar mass itself is dropping, thereby continuously lowering its mass-loss rate. In other words, when we account for the time evolution of a star (basically integrating the mass-loss rate over time), one observes a certain "softening" or "convergence" of the drop in mass with time for various initial masses, as depicted in Fig. 3. Independent of the initial mass, the mass after 2 Myrs is of order $200 M_{\odot}$. This behaviour is similar to that found in Yungelson et al. (2008), but their mass-loss prescriptions were entirely ad-hoc, whilst Fig. 3 of the current study shows an (arguably still simplified) evolution that is based on actual mass-loss computations, albeit at constant effective temperature.

Figure 3 also shows that from current VMS observations (e.g. Schneider et al. 2018), it is not possible to infer the quantitative value of the upper-mass limit. In fact, it is not possible to determine this value quantitatively, until we know both the 
mass-loss history ${ }^{1}$, as well as the mass-accretion rate during star formation.

An important aspect will be to properly investigate if VMS spend enough time at these cool temperatures to have a significant quantitative effect on the upper-mass limit. At the moment we do not yet understand massive star structure \& evolution sufficiently well to be confident in our current evolutionary models. Not only is massive star evolution strongly dependent on $Z$, but the basic question of whether inflated envelopes are stable, or perhaps removed by mass loss, is still unresolved. Ultimately, final answers will depend on the physics of stellar envelopes and winds, both individually, as well as via their combined effect. On the positive side, what we can conclude already is that independent of whether the mass-accretion rate is $Z$-dependent - the upper-mass limit is expected to be $Z$-dependent due to the intrinsic nature of line-opacity mediated radiation-driven winds.

\section{VMS self-enrichment of globular clusters}

Over the past decade, two of the main contenders for the selfenrichment of globular clusters have been massive AGB stars (e.g. D'Ercole et al. 2010) and as an alternative some form of "massive stars". One of the main attractions of AGB stars over massive stars was that AGB stars have slow winds, whilst the line-driven winds of massive O-type stars are fast, up to 2000$3000 \mathrm{~km} \mathrm{~s}^{-1}$. They are so fast, that the polluted wind material cannot be kept within the potential wells of either currently observed or young globular clusters ${ }^{2}$. This is probably one of the main reasons why alternative massive star scenarios, such as the rapidly rotating massive stars (Decressin et al. 2007), massive binaries (de Mink et al. 2009), and red supergiants (Szécsi et al. 2018), have been considered. All these scenarios of course have their pros and cons and for an extensive overview see Bastian \& Lardo (2018) and Gratton et al. (2004).

One of the main problems for all these scenarios is the socalled mass-budget problem and Gieles et al. (2018) recently proposed that a good way around it is the presence of a SMS working as a "conveyer belt" continuously accreting fresh material \& polluting the cluster with enriched material. Although this is a very attractive scenario, the estimated wind velocities of $1000 \mathrm{~km} \mathrm{~s}^{-1}$ are too large in comparison to their estimated escape velocities at the centres of young globular clusters of order $500 \mathrm{~km} \mathrm{~s}^{-1}$ for a cluster mass of $10^{6} M_{\odot}$ (Gieles et al. 2018). Moreover, SMS are still a hypothetical concept whilst VMS, at least up to 200-300 $M_{\odot}$ do exist (Crowther et al. 2010, 2016; Bestenlehner et al. 2011; Oskinova et al. 2013; Vink et al. 2015; Martins 2015). Furthermore, there appears to be more of them than expected from a Salpeter IMF (Schneider et al. 2018) with enhanced mass-loss rates, probably enabling us to overcome the mass budget problem (see Introduction).

It thus appears to be appropriate to consider VMS as the main culprit polluting globular clusters as they dominate the wind feedback (Doran et al. 2013). The structure of VMS involves the inflation of the outer envelope due to the Fe opacity and the proximity to the Eddington limit (Ishii et al. 1999; Gräfener et al. 2012; Sanyal et al. 2015). This implies stellar effective temperatures lower than the usual $40-50 \mathrm{kK}$ ZAMS as discussed

\footnotetext{
1 The mass-loss history will depend on the combined effects of VMS structure and evolution as well as the mass-loss rate.

2 In addition to these intrinsically slower winds, the winds may shock and collide, leading to slow outflows that may be retained in the potential wells of globular clusters, allowing for a second epoch of star formation (Wünsch et al. 2017; Lochhaas \& Thompson 2017).
}

in the current paper. These cooler massive B supergiants have slower winds, from which the wind material may be expected to be maintained in the potential wells of globular clusters. B supergiants as discussed here have the advantage over red supergiants (RSGs) as recently discussed by Szécsi et al. (2018) of being present in the observable universe, for example, as LBVs (Humphreys \& Davidson 1994), whilst RSGs are not known to exist above the Humphreys-Davidson limit.

Although the details of our proposed scenario need to be worked out in terms of self-consistent VMS structure \& evolutionary models, the fact that they must have played a role seems hard to deny, given that they almost certainly existed, whilst alternative scenarios involving either very rapidly rotating stars, RSGs, and possibly even SMS may remain largely hypothetical. The existence of massive binaries in globular clusters is also not yet established, but appears less exotic.

Finally, one of the main reasons for the popularity of the fastrotating massive stars scenario of Decressin et al. (2007) was that it combined two aspects of rapid rotation: (i) it could transport the H-burning core material to the outer layers, whilst rotationally supported disk winds would pollute the globular cluster at low speeds. The issue is that rapid rotation seems empirically rather rare, especially at relatively high $Z$ (see Ramírez-Agudelo et al. 2013). By contrast, VMS form the natural extension of massive stars, and as we have shown here, they are expected to have relatively slow winds.

Finally, rotation-induced mixing may no longer be required for the highest masses, as VMS have both large convective cores and vigorous mass loss. This ensures that their evolution is close to chemically homogeneous (Gräfener et al. 2011; Yusof et al. 2013; Hirschi 2015; Vink et al. 2015), regardless of the stellar rotation rate (Vink \& Harries 2017).

\section{Summary}

We present mass-loss predictions from Monte Carlo radiative transfer models for cool VMS in the $10^{2}-10^{3} M_{\odot}$ range, and we find the following:

- The mass-loss rate is expressed as a function of mass and $Z$ through multiple linear regression.

- We find $\log \dot{M}=-9.13+2.1 \log \left(M / M_{\odot}\right)+0.74 \log \left(Z / Z_{\odot}\right)$, derived for $\left(Z / Z_{\odot}\right)=1-10^{-2}$ and the range $100-900 M_{\odot}$.

- We predict mass-loss rates that rival mass-accretion rates of $10^{-3} M_{\odot} \mathrm{yr}^{-1}$ during massive-star formation, with important consequences for the stellar upper-mass limit.

- We predict relatively slow terminal wind velocities $\left(v_{\infty}\right)$ in the range $100-500 \mathrm{~km} \mathrm{~s}^{-1}$

- We propose slow winds from VMS as a source of chemical pollution of globular clusters.

Acknowledgements. We would like to thank the anonymous referee for constructive questions and comments that helped improve the paper.

\section{References}

Abbott, D. C. 1982, ApJ, 259, 282

Abbott, D. C., \& Lucy, L. B. 1985, ApJ, 288, 679

Anders, E., \& Grevesse, N. 1989, Geochim. Cosmochim. Acta, 53, 197 Banerjee, S., Kroupa, P., \& Oh, S. 2012, MNRAS, 426, 1416

Bastian, N., \& Lardo, C. 2018, ARA\&A, in press [arXiv: 1712 . 01286]

Belczynski, K., Bulik, T., Fryer, C. L., et al. 2010, ApJ, 714, 1217

Belkus, H., Van Bever, J., \& Vanbeveren, D. 2007, ApJ, 659, 1576

Bestenlehner, J. M., Vink, J. S., Gräfener, G., et al. 2011, A\&A, 530, L14 Bestenlehner, J. M., Gräfener, G., Vink, J. S., et al. 2014, A\&A, 570, A38 Cassata, P., Le Fèvre, O., Charlot, S., et al. 2013, A\&A, 556, A68 Castor, J., Abbott, D. C., \& Klein, R. I. 1975, ApJ, 195, 157 
Crowther, P. A., Schnurr, O., Hirschi, R., et al. 2010, MNRAS, 408, 731

Crowther, P. A., Caballero-Nieves, S. M., Bostroem, K. A., et al. 2016, MNRAS, 458,624

de Koter, A., Schmutz, W., \& Lamers, H. J. G. L. M. 1993, A\&A, 277, 561 de Mink, S. E., Pols, O. R., Langer, N., \& Izzard, R. G. 2009, A\&A, 507, L1 de Marchi, G., Panagia, N., Romaniello, M., et al. 2011, ApJ, 740, 11

Decressin, T., Meynet, G., Charbonnel, C., Prantzos, N., \& Ekström, S. 2007, A\&A, 464, 1029

Denissenkov, P. A., \& Hartwick, F. D. A. 2014, MNRAS, 437, L21

Doran, E. I., Crowther, P. A., de Koter, A., et al. 2013, A\&A, 558, A134

D'Ercole, A., D'Antona, F., Ventura, P., Vesperini, E., \& McMillan, S. L. W. 2010, MNRAS, 407, 854

Eldridge, J. J., \& Vink, J. S. 2006, A\&A, 452, 295

Evans, C. J., Taylor, W. D., Hénault-Brunet, V., et al. 2011, A\&A, 530, A108

Figer, D. F. 2005, Nature, 434, 192

Gieles, M., Charbonnel, C., Krause, M. G. H., et al. 2018, MNRAS, 478, 2461

Gräfener, G., \& Hamann, W.-R. 2008, A\&A, 482, 945

Gräfener, G., \& Vink, J. S. 2015, A\&A, 578, L2

Gräfener, G., Vink, J. S., de Koter, A., \& Langer, N. 2011, A\&A, 535, A56

Gräfener, G., Owocki, S. P., \& Vink, J. S. 2012, A\&A, 538, A40

Gratton, R., Sneden, C., \& Carretta, E. 2004, ARA\&A, 42, 385

Hamann, W.-R., Feldmeier, A., \& Oskinova, L. M. 2008, Clumping in Hot-Star Winds Conference (Postdam: University-Verlag), 30

Hillier, D. J. 1991, A\&A, 247, 455

Hirschi, R. 2015, Very Massive Stars in the Local Universe, 412, 157

Hosokawa, T., \& Omukai, K. 2009, ApJ, 691, 823

Humphreys, R. M., \& Davidson, K. 1994, PASP, 106, 1025

Ishii, M., Ueno, M., \& Kato, M. 1999, PASJ, 51, 417

Jiang, Y.-F., Cantiello, M., Bildsten, L., Quataert, E., \& Blaes, O. 2015, ApJ, 813, 74

Kalari, V. M., \& Vink, J. S. 2015, ApJ, 800, 113

Kato, M. 1986, Ap\&SS, 119, 57

Köhler, K., Langer, N., de Koter, A., et al. 2015, A\&A, 573, A71

Krtička, J., Kubát, J., \& Krtičková I. 2016, A\&A, 593, A101

Krumholz, M. R. 2015, Astrophys. Space Sci. Lib., 412, 43

Krumholz, M. R., Klein, R. I., McKee, C. F., Offner, S. S. R., \& Cunningham, A. J. 2009, Science, 323, 754

Kudritzki, R. P. 2002, ApJ, 577, 389

Kuiper, R., Klahr, H., Beuther, H., \& Henning, T. 2010, ApJ, 722, 1556

Kurucz, R. L., \& Bell, B. 1995 Atomic Line Data Kurucz CD-ROM No. 23 (Cambridge, MA: Smithsonian Astrophysical Observatory)

Larson, R. B., \& Starrfield, S. 1971, A\&A, 13, 190

Lochhaas, C., \& Thompson, T. A. 2017, MNRAS, 470, 977

Lucy, L. B., \& Solomon, P. M. 1970, ApJ, 159, 879

Martins, F. 2015, Astrophys. Space Sci. Lib., 412, 9

Moffat, A. F. J., \& Robert, C. 1994, ApJ, 421, 310

Muijres, L. E., de Koter, A., Vink, J. S., et al. 2011, A\&A, 526, A32
Muijres, L. E., Vink, J. S., de Koter, A., Müller, P. E., \& Langer, N. 2012, A\&A, 537, A37

Müller, P. E., \& Vink, J. S. 2008, A\&A, 492, 493

Müller, P. E., \& Vink, J. S. 2014, A\&A, 564, 57

Oskinova, L. M., Steinke, M., Hamann, W.-R., et al. 2013, MNRAS, 436, 3357

Owocki, S. P. 2015, Astrophys. Space Sci. Lib., 412, 113

Pauldrach, A. W. A., \& Puls, J. 1990, A\&A, 237, 409

Pauldrach, A. W. A., Puls, J., \& Kudritzki, R. P. 1986, A\&A 164, 86

Pauldrach, A. W. A., Vanbeveren, D., \& Hoffmann, T. L. 2012, A\&A, 538, A75

Petrov, B., Vink, J. S., \& Gräfener, G. 2016, MNRAS, 458, 1999

Portegies Zwart, S. F., Baumgardt, H., Hut, P., Makino, J., \& McMillan, S. L. W. 2004, Nature, 428, 724

Portegies Zwart, S. F., McMillan, S. L. W., \& Gieles, M. 2010, ARA\&A, 48, 431

Prantzos, N., \& Charbonnel, C. 2006, A\&A, 458, 135

Puls, J., Vink, J. S., \& Najarro, F. 2008, A\&ARv, 16, 209

Ramírez-Agudelo, O. H., Simón-Díaz, S., Sana, H., et al. 2013, A\&A, 560, A29

Ramírez-Agudelo, O. H., Sana, H., de Koter, A., et al. 2017, A\&A, 600, A81

Rosen, A. L., Krumholz, M. R., McKee, C. F., \& Klein, R. I. 2016, MNRAS, 463, 2553

Sanyal, D., Grassitelli, L., Langer, N., \& Bestenlehner, J. M. 2015, A\&A, 580, A20

Schneider, F. R. N., Sana, H., Evans, C. J., et al. 2018, Science, 359, 69

Shaviv, N. J. 2000, ApJ, 532, L137

Smith, N., \& Owocki, S. P. 2006, ApJ, 645, L45

Sobral, D., Matthee, J., Darvish, B., et al. 2015, ApJ, 808, 139

Schmutz, W. 1991, in "Stellar Atmospheres: Beyond Classical Models", eds. Crivellari, L., Hubeny, I., \& Hummer, D. G., NATO ASI Series C, 341, 191

Szécsi, D., Mackey, J., \& Langer, N. 2018, A\&A, 612, A55

Tanaka, K. E. I., Tan, J. C., \& Zhang, Y. 2017, ApJ, 835, 32

van Marle, A. J., Owocki, S. P., \& Shaviv, N. J. 2008, MNRAS, 389, 1353

Vink, J. S. 2012, Astrophys. Space Sci. Lib., 384, 221

Vink, J. S. 2015, Astrophys. Space Sci. Lib., 412, 77

Vink, J. S., \& Gräfener, G. 2012, ApJ, 751, L34

Vink, J. S., \& Harries, T. J. 2017, A\&A, 603, A120

Vink J. S., de Koter A., \& Lamers H. J. G. L. M. 1999, A\&A 345, 109

Vink, J. S., de Koter, A., \& Lamers, H. J. G. L. M. 2000, A\&A 362, 295

Vink, J. S., de Koter, A., \& Lamers, H. J. G. L. M. 2001, A\&A 369, 574

Vink, J. S., Muijres, L. E., Anthonisse, B., et al. 2011, A\&A 531, A132

Vink, J. S., Heger, A., Krumholz, M. R., et al. 2015, Highlights Astron., 16, 51

Vink, J. S., Evans, C. J., Bestenlehner, J., et al. 2017, IAU Symp., 329, 279

Weidner, C., \& Kroupa, P. 2004, MNRAS, 348, 187

Wolfire, M. G., \& Cassinelli, J. P. 1987, ApJ, 319, 850

Woosley, S. E., \& Heger, A. 2015, Astrophys. Space Sci. Lib., 412, 199

Wünsch, R., Palouš, J., Tenorio-Tagle, G., \& Ehlerová, S. 2017, ApJ, 835, 60

Yorke, H. W., \& Sonnhalter, C. 2002, ApJ, 569, 846

Yungelson, L. R., van den Heuvel, E. P. J., Vink, J. S., Portegies Zwart, S. F., \& de Koter, A. 2008, A\&A, 477, 223

Yusof, N., Hirschi, R., Meynet, G., et al. 2013, MNRAS, 433, 1114 